\title{
DEEP LEARNING APPROACHES FOR HUMAN ACTIVITY RECOGNITION USING WEARABLE TECHNOLOGY
}

\author{
PRISTUPI DUBOKOG UČENJA ZA PREPOZNAVANJE \\ AKTIVNOSTI LJUDI PRIMENOM ODEVNE TEHNOLOGIJE
}

\author{
Milica Janković ${ }^{1}$, Andrej Savić ${ }^{1,2}$, Marija Novičić ${ }^{1}$, Mirjana Popović ${ }^{1}$
}

${ }^{1}$ University of Belgrade, School of Electrical Engineering, Belgrade, Serbia

${ }^{2}$ Tecnalia Serbia; Belgrade, Serbia

Correspondence: piperski@etf.rs

\section{Abstract}

The need for long-term monitoring of individuals in their natural environment has initiated the development of a various number of wearable healthcare sensors for a wide range of applications: medical monitoring in clinical or home environments, physical activity assessment of athletes and recreators, baby monitoring in maternity hospitals and homes etc. Neural networks (NN) are data-driven type of modelling. Neural networks learn from experience, without knowledge about the model of phenomenon, but knowing the desired „output" data for the training ,input” data. The most promising concept of machine learning that

Keywords:

deep learning,

human activity monitoring, human machine interface, wearable sensors, smart sensors, multimodal interface involves NN is the deep learning (DL) approach. The focus of this review is on approaches of DL for physiological activity recognition or human movement analysis purposes, using wearable technologies. This review shows that deep learning techniques are useful tools for health condition prediction or overall monitoring of data, streamed by wearable systems. Despite the considerable progress and wide field of applications, there are still some limitations and room for improvement of DL approaches for wearable healthcare systems, which may lead to more robust and reliable technology for personalized healthcare.
Janković M. et al. MedPodml 2018, 69(3):14-24 (c) The authors declare no conflicts of interest. doi: $10.5937 / \mathrm{mp} 69-18039$

Editorial board: podmladak.med.bg@gmail.com e-ISSN: 2466-5525 
Sažetak

Ključne reči:

duboko učenje, praćenje ljudske aktivnosti, čovek-mašina interfejs, odevni senzori, pametni senzori, multimodalni interfejs
Potreba za dugotrajnim praćenjem osoba u njihovom svakodnevnom okruženju inicirala je razvoj velikog broja nosivih senzora (integrisanih u delove garderobe) s različitom primenom, kao što su medicinsko praćenje u kliničkim i kućnim uslovima, procena fizičke aktivnosti sportista i rekreativaca, praćenje beba u porodilištima i kućama i sl. Neuralne mreže (NM) predstavljaju tip modelovanja zasnovan na velikom broju podataka. Ove mreže uče na osnovu iskustva, bez poznavanja modela fenomena, ali znajući šta su željeni „izlazni” podaci za obučavajuće „ulazne” podatke. Koncept mašinskog učenja koji najviše obećava i uključuje NM jeste duboko učenje (DU). Fokus ovog preglednog rada je u pristupima DU u cilju prepoznavanja fizioloških aktivnosti i analize ljudskih pokreta primenom "odevne tehnologije". Ovaj rad pokazuje da su tehnike dubokog učenja korisne alatke za predikciju zdravstvenih stanja ili celokupno praćenje podataka koji se šalju sa "odevnih" senzora. Uprkos značajnom napretku i obećavajućoj oblasti primene, i dalje postoje ograničenja i prostor za unapređenje pristupa DU za "odevne" zdravstvene sisteme koji će dovesti do njihove pouzdane primene i omogućiti personalizovanu zdravstvenu zaštitu.

\section{Introduction}

In the last two decades, the development of technology and computer science has enabled the miniaturization of electronic components and sensors, their integration into clothes or jewelry for continuous healthcare or behavior monitoring and sharing acquired data using Internet of Things (IoT) concepts (Figure 1) (1-3). Wearable healthcare devices integrate: 1) sensor with analog front-end (conditioning circuitry); 2) microcontroller unit for data acquisition; 3 ) wireless module for data transmission (Bluetooth, ZigBee, GPRS, GSM etc.); and 4) battery cell for independent power supply of the device. Acquired data could be stored on the local medium (SD memory card), the local computer device (laptop, tablet, smartphone) or on the secured cloud. Basic non-compliant processing could be performed by AFE and microcontroller in the wearable device, but more complex data science (advanced signal processing, data mining, machine learning, decision support etc.) are usually performed offline.

The results of measuring by wearable devices could be used for monitoring of vital parameters of the human body, improving diagnostics by medical experts, or as a feedback information for the user.

The need for long-term monitoring of individuals in their natural environment has initiated the development of a various number of wearable sensors for a wide range of applications: medical monitoring in clinical or

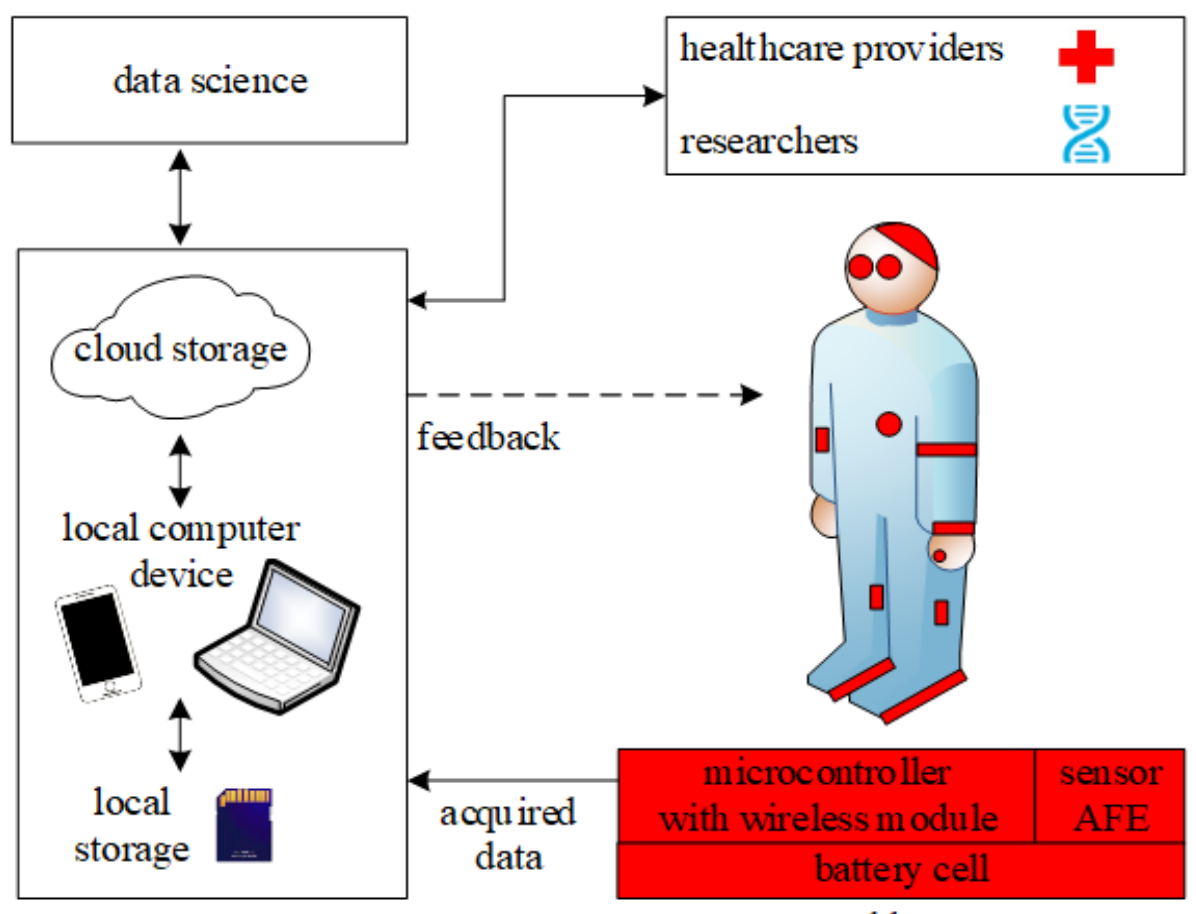

wearable sensor

Figure 1. Wearable system - data acquisition and transmission concept, AFE - analog front-end 
home environments, physical activity assessment of athletes and recreators, baby monitoring in maternity hospitals and homes (4). Wearable technology combines several disciplines: electronics, biomedical engineering, fashion, smart textile and flexible-stretchable-printable electronics, with the aim to design unobtrusive sensing devices for human activity monitoring $(5,6)$. Also, an emerging trend of simultaneous recording using different wearable devices, so-called multimodal interfacing, is present in the literature (7).

Examples of various wearable devices developed by research university teams and R\&D companies are presented in Figure 2. Paradiso et al. (8) produced a Wearable Wellness System (WWS) for real-time monitoring of 1-lead ECG (electrocardiogram) and respiration rate. Textile ECG electrodes are placed on the thorax, and textile piezoresistive sensor (breathing sensor) is placed above them in the middle (9). Emotiv EPOC ${ }^{\bullet}$ neuroheadset is a wearable 14-channel EEG system, validated as brain computer interface (BCI) device $(10,11)$, used in the research of event-related potential (ERP) studies $(12,13)$ and emotion recognition experiments $(14,15)$. Myo armband ${ }^{\oplus}$ is a hand gesture controller that integrates eight segments with electromyography (EMG) sensors (each segment includes one EMG amplifier with bar electrodes), and one segment additionally includes a three-axis accelerometer and a three-axis gyroscope (16). This device could be used as a substitution of computer mouse, providing individuals without hand or forearm to make human- computer control, but also supports other applications of humanmachine interface. Djurić-Jovičić et al. (17) developed SENSY system of interactive shoes inertial measurement units (IMU) that measure force distribution and stride parameters in gait analysis. Moodmetric ${ }^{\oplus}$ ring measures electro dermal activity of the skin, as an indicator of sympathetic activation (18) and the appropriate application, estimates the level of physiological and psychological arousal (19).

Mobile eye tracking devices in the form of ordinary glasses frames, with storing and real-time processing capabilities, have expanded the research and the application field of gaze gestures in daily life environment like (20).

Development of wearable minimally-invasive glucose monitor sensors has made the revolution in continuous diabetes treatment and significantly improved the quality of life of diabetics (21). Such wearable sensors insert a needle in the subcutaneous tissue of the abdomen or the arm and measures in real-time (every 1-5 min) the electrical current of glucose-oxidase reaction, proportional to the glucose concentration. Zheng et al. developed a prototype of armband for 24-hour blood pressure (BP) monitoring that indirectly measure BP using pulse transit time obtained from ECG and photoplethysmogram (PPG) (22).
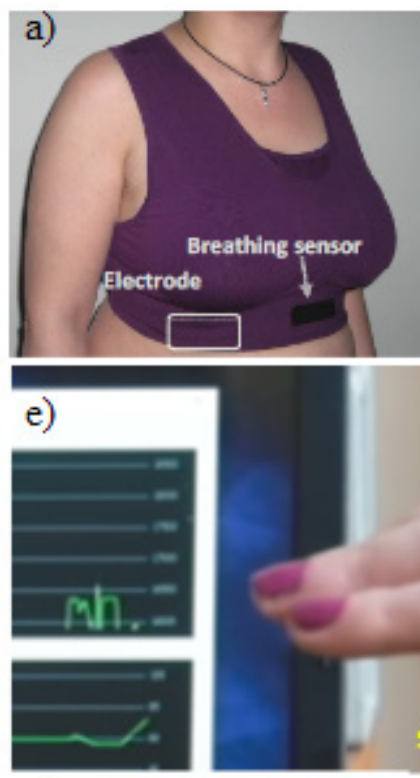

g)
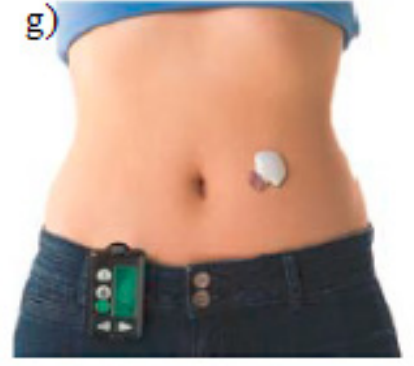
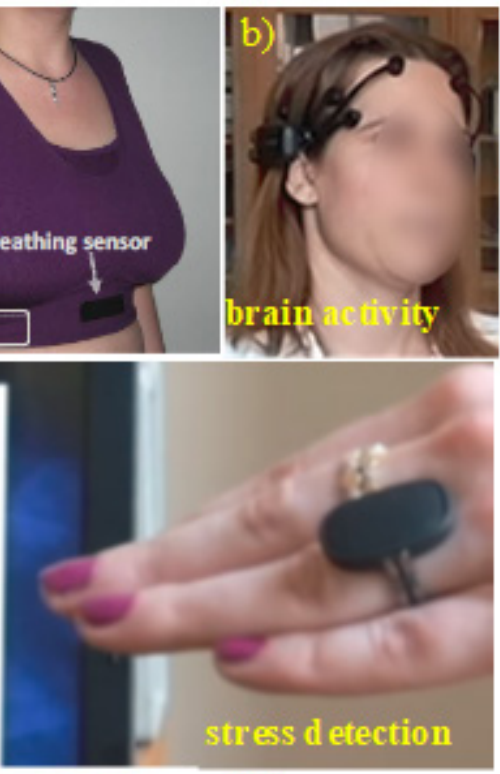

h)

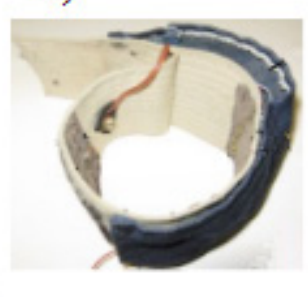

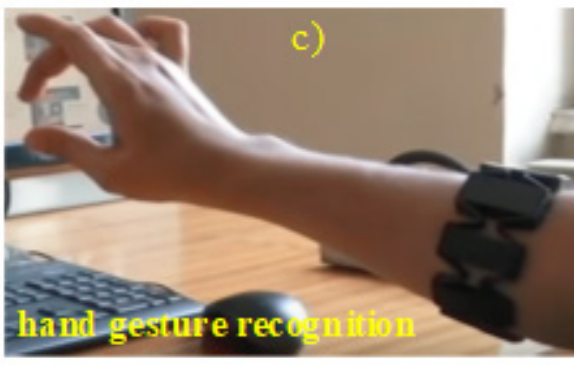

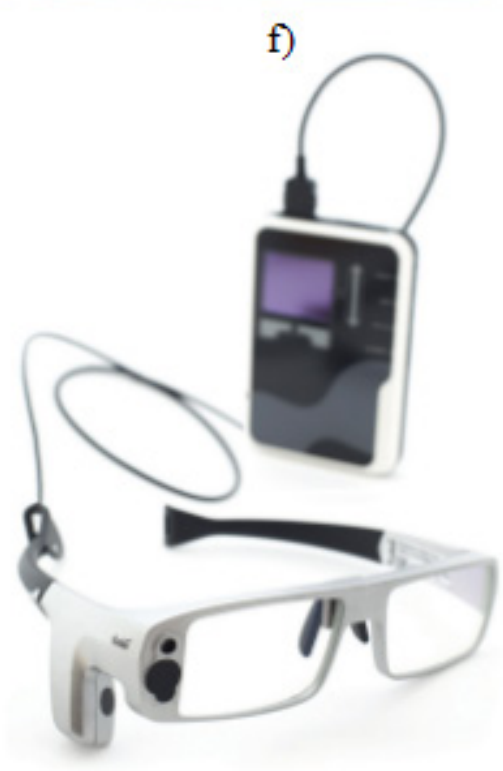

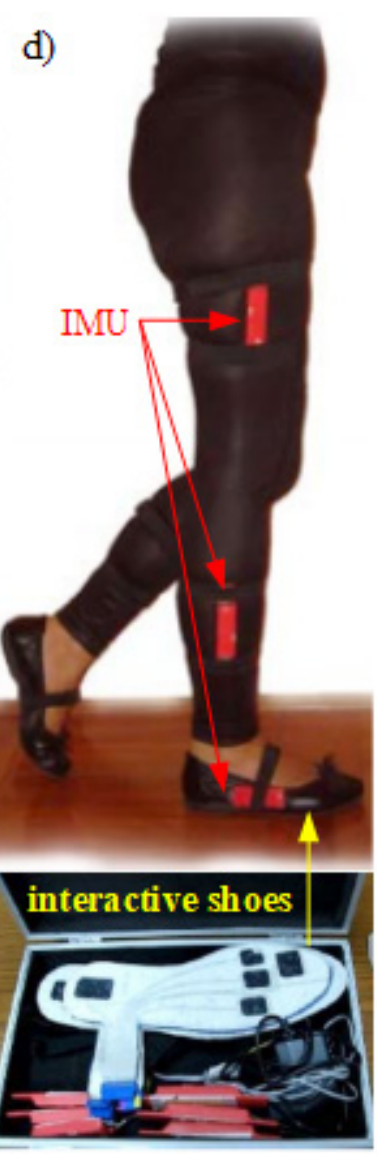

Figure 2. a) Wearable Wellness System from (8), b) Emotiv EPOC ${ }^{\circledast}$ neuroheadset, c) Myo armband ${ }^{\circledR}$ hand gesture controller, d) SENSY system for gait analysis (17), e) Moodmetric ${ }^{\circ}$ ring for stress follow, f) Tobii ${ }^{\circledR}$ eye tracker glasses form (20), g) Medtronic ${ }^{\oplus}$ continuous glucose monitor form (21), h) A prototype of wearable 24-hour blood pressure device form (22) 
Deep Learning algorithms

Data science is a "concept to unify statistics, data analysis, machine learning and their related methods" in order to "understand and analyze actual phenomena" with data (23). Machine learning (ML) is an application of artificial intelligence (AI) that provides systems the ability to automatically learn and act like humans do, based on experience rather than based on strictly defined algorithms. An individual measurable representation of a phenomenon being observed is called a feature (24). The performance of ML algorithms depends on the features that are chosen, and this is the most important step for the various ML algorithms.

One of the growing fields of ML is Deep Learning (DL). It solves the main issue of ML by expressing complex representation in terms of other, simpler representations, allowing the computer to build complex concept out of simpler concepts (25). Principles of several deep learning algorithms: feedforward neural networks (FNN), convolutional neural networks $(\mathrm{CNN})$ and recurrent neural networks (RNN) will be briefly explained in this chapter. The data processing in those artificial neural networks $(\mathrm{NN})$ is very similar to decision making procedure in biological neural structure. Neural networks learn from experience without knowledge about the model of phenomenon but knowing what the desired reaction is ("output" data) for the appropriate training "input" data. Based on the given input/output data, they develop their own knowledge, which allows them to make conclusions during testing on new datasets.

The basic NN unit is a perceptron or artificial neuron, Figure 3. The perceptron combines several inputs using and produces a single output. It consists of two parts: net function and activation function. Net function summarizes information from $m$ external sources or other neurons. Net function is a linear combination of products of inputs $\mathrm{x} \_\mathrm{i}$ and weight coefficients w_i, Eq. (1):

$n=w_{0}+w_{1} x_{1}+w_{2} x_{2}+\cdots+w_{m} x_{m}, i \in[1, \mathrm{~m}]$.

The activation function (threshold, linear, sigmoid etc.) is applied to the output of net function. The output value of the perceptron is defined as the output of activation function.

Feedforward deep learning is based on feedforward neural networks (multi-layered perceptron - MLP). These neural networks (Figure 4) consist of large number of highly connected perceptron grouped into layers (26). The number of layers defines depth of neural network. Number of neurons in one layer defines width of model. First layer is called input layer and the last one is called output layer. Layers between input and output layer are called hidden layers. These networks are called feedforward because the information flows from the input layer, through hidden layers, to the output layer, which means that there is no feedback from the output to the input. During the training phase, learning algorithm updates weight factors depending on the difference between current outputs and desired outputs and when the difference becomes satisfactory small, learning algorithm stops. Estimated weight factors are stored for the testing phase. During the training phase, over fitting should be avoided and $\mathrm{NN}$ must generalize well with unknown inputs too. Autoencoder is a type of NN where the number of input and output nodes is the same. Deep Belief Network (DBN) is composed of layers of Restricted Boltzmann Machines (RBMs) for the pre-train phase and then a feed-forward network for the fine-tune phase.

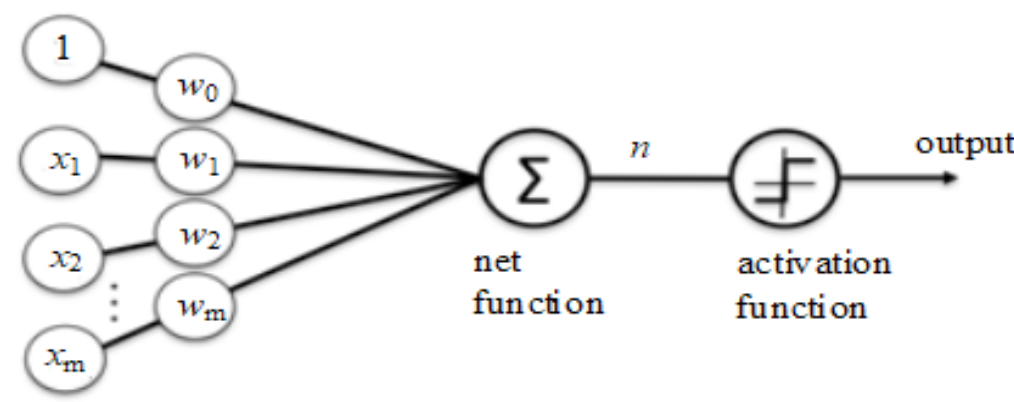

Figure 3. A description of how perceptron (artificial neuron) works

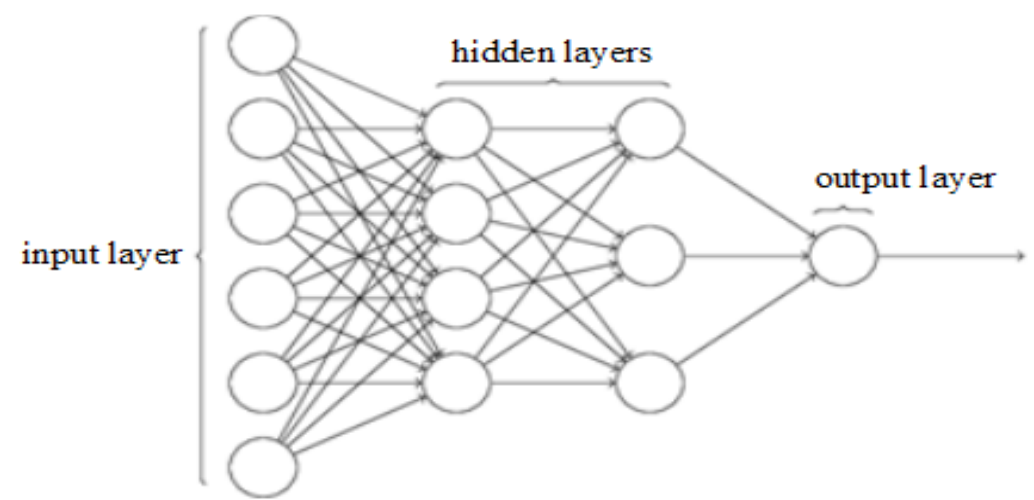

Figure 4. An example of feedforward neural network 
Janković M. et al. Deep learning approaches for human activity recognition using wearable technology. MedPodml 2018, 69(3):14-24

Convolutional neural networks $(\mathrm{CNN})$ are specialized neural networks for processing data that has grid-like topology (time-series data or images). They have sparse interactions, which is accomplished by using the kernel smaller than the input (27). For example, if the input data have thousands or millions of samples, local features could be detected using kernels that occupies only a dozen or a hundred of samples. Efficiency of CNN is improved compared to FNN and memory requirements are reduced. Using classic FNN, an input neuron affects all outputs neurons, while in $\mathrm{CNN}$ an input neuron only affects several output neurons (number is defined by kernel width). The typical layer of CNN consists of three stages (Figure 5). The first stage performs several convolutions in parallel to produce linear activation. In the second, detector stage, each linear activation is run through nonlinear activation function. The last, third stage uses pooling function to make the output invariant to the noise and disorder.

The best performances of $\mathrm{CNN}$ are achieved for two-dimensional image topology, while for processing of one-dimensional time series data, it is best to use RNN. Each output of RNN depends on the previous outputs (28), Figure 6. RNN shares their weight factors through a very deep computation graph. Long Short Term Memory (LSTM) is a type of RNN that uses special units to include a 'memory cell' that can maintain information in memory for long periods of time.

\section{Deep Learning applications in wearable healthcare systems}

Several comprehensive reviews about the rise of wearable sensors and the significance of using data science methods for improving wearable healthcare information systems could be found in the literature (29-32). They emphasize the increasing trend of continual multimodal sensing for early diagnosis of diseases, prevention of chronic conditions, quick response in emergencies, physiological activity monitoring. Gravina et al. (33) have published a global overview about multi-sensor fusion of information in body sensor networks. Banaee et al. (34) presented the data mining review for healthcare and wearable sensors for the following vital signals: electrocardiogram, heart rate, blood pressure, respiratory rate, oxygen saturation, photoplethysmography and blood glucose. In this chapter, we will focus on approaches of DL for physiological activity recognition or human movement analysis purposes. The general concept of the DL approach for wearable sensor data is presented in Figure 7. The starting point in DL approaches is the acquisition of raw data from different wearable sensors. The next step is data preprocessing (filtering, de-noising, normalization, all data synchronization etc.) followed by the feature extraction (in time or frequency domain or nonlinear features) and the selection of the most informative features for DL training/testing. The results of feature extraction and selection and additional

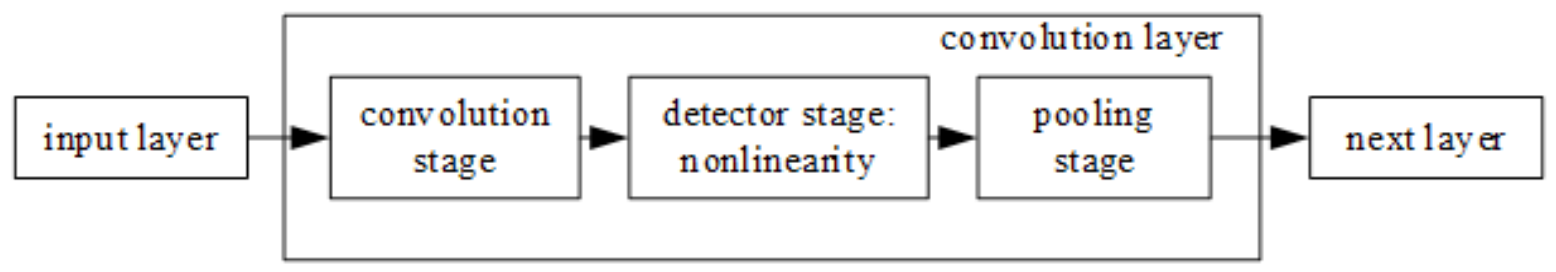

Figure 5. The structure of convolutional neural network

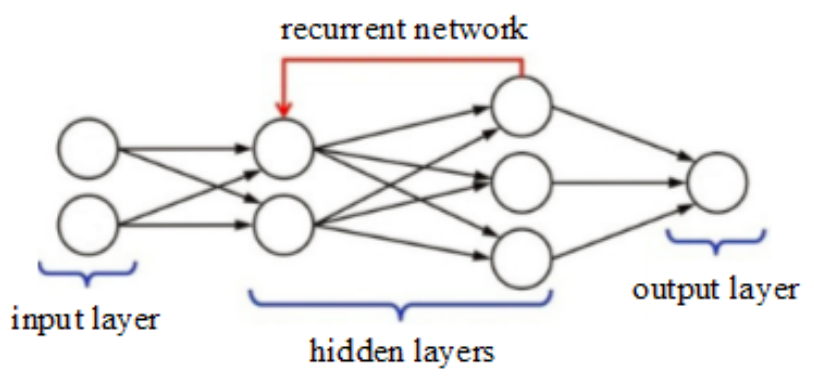

Figure 6. The structure of recurrent neural network

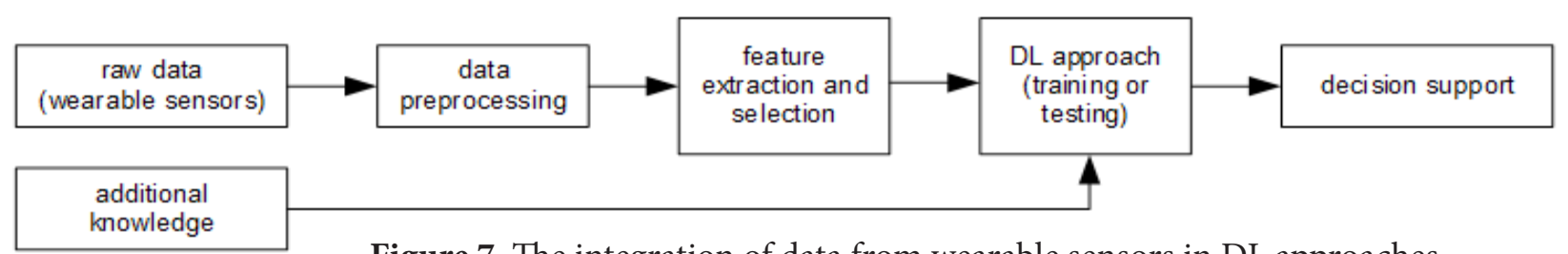

Figure 7. The integration of data from wearable sensors in DL approaches 
knowledge (expert opinion, patient metadata, study metadata etc.) are inputs for training/testing DL algorithm that will finally make a decision about the medical phenomenon of interest (detection, prediction etc.).

The summary of possible DL applications in wearable healthcare approaches is presented in Table 1. with the comparison of their accuracy.

Sarkar et al. (35) proposed a framework utilizing deep learning that recognizes human cognitive activities in real time via fusion of multiple EEG sensors in an unconstrained environment and selects a smaller sensor suite appropriate for wearable systems. Classification performances for two major activities "listening" and "watching" was high using DBN and CNN approaches $(91.15 \%$ and 91.63\%, respectively).
Langkvist et al. (36) tested a two-layer DBN with 200 hidden units in both layers. They report that the DBN method increased the accuracy of sleep scoring $(91.33 \%)$ by approximately 3\%, when compared with the manual methods. Authors concluded that, for multimodal data, it is favorable to utilize separate DBNs for each of the signals and then combine their outputs with a secondary DBN.

Zhang et al. (37) applied the sparse version of DBN (SDBN) for sleep stage classification. They also used a voting principle based on classification entropy using SDBN and combination of classifiers including Support Vector Machine (SVM), k-nearest neighbors (KNN) and Hidden Markov Model (HMM), attaining 91.31\% accuracy.

Dong et al. (38) have used LST network for sequential data learning to optimize classification performance

Table 1. Summary of different deep learning methods possible for wearable healthcare. Acc - accuracy, $\mathrm{P}$ - precision, BA - Bland Altman slope (systematic error)

\begin{tabular}{|c|c|c|c|c|}
\hline Data & Application & Network type & & Results \\
\hline \multirow[t]{9}{*}{ EEG } & \multirow[t]{2}{*}{ Cognitive activity recognition } & $\mathrm{DBN}$ & 35 & Acc: $91.15 \%$ \\
\hline & & $\mathrm{CNN}$ & & Acc: $91.63 \%$ \\
\hline & \multirow[t]{3}{*}{ Sleep stage scoring } & $\mathrm{DBN}$ & 36 & Acc: $91.33 \%$ \\
\hline & & & 37 & Acc: $91.31 \%$ \\
\hline & & LSTM & 38 & Acc: $85.92 \%$ \\
\hline & Anomaly detection & $\mathrm{DBN}$ & 39 & $\begin{array}{l}\text { P: } 0.1920 \\
\text { high performance }\end{array}$ \\
\hline & \multirow[t]{2}{*}{$\begin{array}{l}\text { Classification of motor } \\
\text { imagery }\end{array}$} & $\begin{array}{l}\text { CNN } \\
\text { Autoencoder }\end{array}$ & 40 & Acc: $77.6 \%$ \\
\hline & & Frequency DBN & 41 & Acc: $84 \%$ \\
\hline & $\begin{array}{l}\text { Motion-onset Visual Evoked } \\
\text { Potential feature extraction }\end{array}$ & $\mathrm{CNN}$ & 42 & Acc: $87.5 \%$ \\
\hline EMG & Hand movement classification & $\mathrm{DBN}$ & 43 & $\begin{array}{l}\text { Acc: } 66.59 \% \\
\text { (healthy) } \\
38.09 \% \text { (amputees) }\end{array}$ \\
\hline \multirow[t]{4}{*}{ ECG } & Arrhythmia classification & $\mathrm{DBN}$ & 45 & Acc: $98.83 \%$ \\
\hline & Abnormal ECG recognition & DNN & 47 & Acc: $85.52 \%$ \\
\hline & Biometric user identification & CNN-LSTM & 48 & Acc: $99.54 \%$ \\
\hline & Diabetes detection & $\mathrm{CNN}$ & 49 & Acc: $95.1 \%$ \\
\hline \multirow[t]{3}{*}{ PPG } & $\begin{array}{l}\text { Monitoring and detecting of } \\
\text { atrial fibrillation }\end{array}$ & $\mathrm{DBN}$ & 50 & Acc: $91.8 \%$ \\
\hline & Biometric user identification & DBN & 51 & Acc: $96 . t 1 \%$ \\
\hline & Blood pressure monitoring & $\begin{array}{l}\text { CNN, LSTM- } \\
\text { RNN }\end{array}$ & 52 & $\begin{aligned} & \text { BA: } 0.47 \text { (systolic) } \\
& 0.16 \text { (diastolic) } \\
&\end{aligned}$ \\
\hline \multirow[t]{6}{*}{ Motion } & Human activity recognition & CNN, LSTM & 53 & Acc: $95.8 \%$ \\
\hline & $\begin{array}{l}\text { Closed loop for human } \\
\text { activity recognition }\end{array}$ & $\mathrm{DBN}$ & 54 & Acc: $90 \%$ \\
\hline & \multirow{3}{*}{$\begin{array}{l}\text { Mobile applications for } \\
\text { activity recognition }\end{array}$} & $\mathrm{DBN}$ & 55 & Acc: $73-94 \%$ \\
\hline & & $\mathrm{CNN}$ & 56 & Acc: $95.75 \%$ \\
\hline & & & 57 & Acc: $91.5-98.2 \%$ \\
\hline & Movement disorder & $\mathrm{CNN}$ & 58 & Acc: $90.9 \%$ \\
\hline
\end{tabular}


with one-channel EEG. Authors have tested single-channel EEG options on the forehead (F4-EOG left and Fp2-EOG left). Evaluation of data from 62 people and 494 hours of sleep demonstrated better performance of their algorithm than existing approaches with vertex or occipital electrode placements. The classification accuracy using LST network was $85.92 \%$ and it was higher than using SVM (79.7\%), Random Forest (RF, 81.67\%) or MLP (81.43\%).

Wulsin et al. (39) applied DBN in a semi-supervised paradigm to model EEG waveforms for classification and anomaly detection i.e. small group of isolated waveform patterns, such as spikes, seizures, eye blinks and other artifacts/noise. Their results show that DBN performance was comparable to standard classifiers (decision trees, SVM, $\mathrm{KNN}$ ), and classification time was found to be 1.7 to 103.7 times faster than the other high-performing classifiers. Authors suggested that DBNs with raw data inputs may be more effective for online automated anomaly recognition in the EEG than other common techniques.

Tabar et al. (40) have used deep learning approach to improve classification of EEG motor imagery for BCI. They employed CNN and Autoencoder network to classify motor imagery from EEG. They proposed a new deep network in which the features that are extracted by CNN are classified through the Autoencoder network. The averaged classification error was $77.6 \%$. They report $9 \%$ improvement over the winner algorithm of $\mathrm{BCI}$ competition IV (Berlin, 2008).

$\mathrm{Lu}$ et al. (41) estimated frequency domain representations of EEG signals for motor imaginary obtained via fast Fourier transform (FFT) and wavelet package decomposition (WPD) to train three RBMs, which were stacked up with an additional output layer to form a four-layer neural network, which is named the Frequential DBN (FDBN). The output layer used Softmax regression for classification and the conjugate gradient method and backpropagation were used to fine tune the FDBN. Results on benchmark datasets, showed statistically significant improvement in classification, using FDBN over other selected state-of-the-art methods ( $84 \%$ vs. $73-80 \%$ ).

$\mathrm{Ma}$ et al. (42) combined DBN with compressed sensing to extract discriminative Motion-onset Visual Evoked Potentials (mVEP) information for improving the BCI performance. The deep learning and compressed sensing approach generated the multi-modality features which improved BCI performance with approximately 3.5\% accuracy incensement. Deep learning and compressed sensing approach yielded higher classification accuracy $(87.5 \%$ vs. $84 \%$ ), against using conventional mVEP features, and were effective for subjects with relatively poor performance.

Atzori et al. (43) used CNN to classify 50 hand movements using surface EMG signals. Authors used Ninapro open database (44) of 78 subjects: 67 health subjects and 11 subjects with trans radial amputee. The average classification accuracy using simple CNN and classical classification methods (SVM, KNN, random forest, linear discriminant analysis) were comparable $(66.59 \%$ vs. $62.06 \%$ for dataset 1 of health subjects, $60.27 \%$ vs. $60.28 \%$ for dataset 2 of health subjects, $38.09 \%$ vs. $38.82 \%$ for amputees).

Yan et al. (45) used standard MIT-BIH database (46) of ECG signals to train RBMs. Half of the data was used for RBMs training, 30\% was used for fine-tuning of DBN and $20 \%$ was used for testing. It is shown that by combining the two ECG-lead the accuracy, sensitivity and specificity reached to $98.83 \%, 99.83 \%$ and $96.05 \%$, respectively.

Ripol et al. (47) compared the efficacy of DBN recognition of abnormal 12-lead ECG in a large group of patients (1390 patients from Hospital Clinic in Barcelona) against several algorithms: SVM, KNN, Extreme Learning Machines (ELM) and professional algorithm of dedicated cardiology system. Both SVM (accuracy $84.76 \%$ and specificity $73.46 \%$ ) and DBN (accuracy $85.52 \%$ and specificity $78.27 \%$ ) resulted by better accuracy and specificity than other methods.

Page et al. (48) applied deep neural network (DNN) on identified QRS segments of 90 subjects in resting state to biometrically identify them. The best results were obtained using a single hidden layer while deeper networks had overfitting problems. The accuracy of applied NN was $99.54 \%$, sensitivity was $99.49 \%$ and specificity was $99.55 \%$.

Swapna et al. (49) applied CNN-LSTM on heart rate extracted from ECG signals to detect diabetes, with the accuracy of $95.1 \%$. This seems to be a promising method for non-invasive detection of diabetes.

Shashikumar et al. (50) performed real-time detection of atrial fibrillation in patients. They used continuous wavelet transform of the PPG signal recorded by Simband smart watch to extract features for training of $\mathrm{CNN}$. The obtained accuracy was $91.8 \%$ and comparable to ECGbased approaches.

Jindal et al. (51) compared the performance of DBN approach with classic KNN and fuzzy classifier in biometric user identification. The DBN approach included the clustering step to subgroups before pre-training by RBMs and DBN fine tuning. The combined clustering and DL method had $96.1 \%$ accuracy in biometric authorization, which was more than $10 \%$ higher than by classic methods.

Ruiz-Rodríguez et al. (52) simultaneously recorded blood pressure invasively (by radial artery catheter) and non-invasively (by photoplethysmograph). The RBM was trained to assess blood pressure using PPG signals in the group of 572 patients with stable blood pressure. A systematic error (Bland-Altman slope) was 0.47 and 0.16 for systolic and diastolic arterial pressure, respectively.

Wearable DL systems for the recognition of physical human activity were considered by different authors. The input signals for DL training in such systems are from motion sensors (accelerometers, gyroscopes). Ordóñez et al. (53) showed that the combination of CNN and RNN was effective in human activity recognition of 18 gesture tasks with the accuracy of $95.8 \%$. The robustness of activity recognition could be improved using closed loop concept introduced by Saeedi et al. (54) and the accuracy of activity recognition could reach $90 \%$. Several authors presented frameworks for activity recognition using mobile devices indicating the possibilities of commercial applications 
(55-57). Bhattacharya et al. (55) presented a simple RBM solution that could work on smart watch processor (Qualcomm Snapdragon 400). The smart watch prototype included following sensors: accelerometer, gyroscope, barometer, magnetometer, light and temperature. Three different RBM architectures were tested to support three types of daily scenarios: gestures, transportation and physical activities, and transition between indoor/outdoor environments. Classification results by RBM method had higher accuracy in comparison to conventional methods (SVC, RF, decision trees). The results of testing the battery life were between 6 and 52 hours, depending on the RBM model. Ronao et al. (56) presented results on the study of 30 volunteers that performed different physical activities (walking, sitting, standing, laying) while the smart phone was in their pockets, collecting accelerometer and gyroscope data. They report the classification accuracy of 95.75\% for human activity recognition by CNN. Also, one solution for $\mathrm{CNN}$ efficient implementation of human activity recognition (walking, jogging, cycling etc.) on a low-power device was introduced by Ravi et al. (57). Eskofier et al. (58) explained the clinical application and superiority of deep CNN in recognition of bradykinesia in Parkinson's patients (based on data from accelerometers positioned on the forearm) in comparison with classic methods (SVM, KNN, PART, Ada Boost M1), 90.9\% vs. $85.6 \%, 67.1 \%, 81.7 \%$ and $86.3 \%$, respectively.

\section{Limitations and future directions}

We have presented the summary of 23 papers with DL methods possible for wearable healthcare application and compared the results of their accuracy. Most of them use DBN or CNN concepts as DL methods, Table 1. Also, we have cited 5 review papers in the wider field of data science methods applied for wearable healthcare.

Deep Learning techniques have high computational complexity, but also high accuracy in various types of applications, because of which they are promising approaches for extraction and/or classification of data in health informatics. However, before their expansion in usage, the following questions should be resolved (59-61): ${ }^{1)}$ standardization of data acquisition, normalization and preprocessing procedures and collecting of huge amount of data for the homogenous groups of subjects related to the phenomenon of interest - this is the key condition for the validity of data driven modelling; ${ }^{2)}$ designing of DL approaches for longitudinal studies; ${ }^{3)}$ developping sequrity models for data acquisition and delivery of results/feedback; ${ }^{4)}$ including expert knowledge in DL process; and ${ }^{5)}$ finding a way to rationalize neural predictions.

Wearable systems for analyzing state of the user have been developed and tested for wide area of application: motion analysis, human machine interaction (HMI), detection (and/or prediction) of different type of seizures, drowsiness monitoring (for example during driving), sleep monitors, emotion recognition, etc. Significant number of studies explore their applicability in the area of neurorehabilitation and feedback and show progress in improving communication, mobility and environment control in severely disabled users. This review shows that DL techniques are useful tools for health condition prediction or overall monitoring of data streamed by wearable systems. Nowadays, DL is present in commercial systems for speech recognition and computer vision and it is expected to have an important role in smart healthcare in the future. It is expected that wearable healthcare systems with DL approaches will be successfully integrated in mobile systems (5264). The direct consequence of the application of such systems, in both home and clinical practice, will be decreasing expenses for home and clinical healthcare, or supervising athletes. Despite the considerable progress, there is still room for improvement of DL approaches for wearable healthcare systems, which will lead to more trustable and reliable application and personalized healthcare.

\section{Acknowledgement}

This research was partly supported by the Ministry for Education, Science and Technology Development of Serbia, Belgrade, Serbia (OS 175016).

\section{References}

1. Rodgers MM, Pai VM, Conroy RS. Recent advances in wearable sensors for health monitoring. IEEE Sensors Journal, 2015; 15(6): 3119-3126.

2. Bonato P. Wearable sensors and systems. IEEE Engineering in Medicine and Biology Magazine. 2010; 29(3): 25-36.

3. VukićevićS, StamenkovićZ, Murugesan S, Bogdanović Z, Radenković B. A new telerehabilitation system based on internet of things. Facta Universitatis, Series: Electronics and Energetics, 2015; 29(3): 395-405.

4. Bloss, R. Wearable sensors bring new benefits to continuous medical monitoring, real time physical activity assessment, baby monitoring and industrial applications. Sensor Review, 2015; 35(2): 141-145.

5. Zheng YL, Ding XR, Poon CCY, Lo BPL, Zhang H, Zhou XL et al. Unobtrusive sensing and wearable devices for health informatics. IEEE Transactions on Biomedical Engineering, 2014; 61(5): 1538-1554.

6. Stoppa M, Chiolerio A. Wearable electronics and smart textiles: a critical review. Sensors. 2014; 14(7): 11957-11992.

7. Kumari P, Mathew L, Syal P. Increasing trend of wearables and multimodal interface for human activity monitoring: A review. Biosensors and Bioelectronics. 2017; 90: 298-307.

8. Paradiso R, Bianchi AM, Lau K, Scilingo EP. PSYCHE: Personalised monitoring systems for care in mental health. In: Annual international conference of the IEEE Engineering in Medicine and Biology Society (EMBC). August 31-September 4, Buenos Aires, Argentina. 2010; p. 3602-3605.

9. Scilingo EP, Gemignani A, Paradiso R, Taccini N, 
Ghelarducci B, De Rossi D. Performance evaluation of sensing fabrics for monitoring physiological and biomechanical variables. IEEE Transactions on information technology in biomedicine. 2005; 9(3): 345-352.

10. Liu Y, Jiang X, Cao T, Wan F, Mak PU, Mak PI et al. Implementation of SSVEP based BCI with Emotiv EPOC. In: IEEE International Conference on Virtual Environments Human-Computer Interfaces and Measurement Systems (VECIMS). 2-4 July, Tianjin, China, 2017; p. 34-37.

11. Taylor GS, Schmidt C. Empirical evaluation of the Emotiv EPOC BCI headset for the detection of mental actions. In: Proceedings of the Human Factors and Ergonomics Society Annual Meeting. 22-26 October, Boston, Massachusetts, USA, 2012; 56(1): 193-197.

12. Duvinage M, Castermans T, Petieau M, Hoellinger T, Cheron G, Dutoit T. Performance of the Emotiv Epoc headset for P300-based applications. Biomedical engineering online. 2013; 12(1): 56.

13. Badcock NA, Mousikou P, Mahajan Y, De Lissa P, Thie J, McArthur G. Validation of the Emotiv EPOC ${ }^{\circ}$ EEG gaming system for measuring research quality auditory ERPs. PeerJ. 2013; 1: e38.

14. Ramirez R, Vamvakousis Z. Detecting emotion from EEG signals using the emotive epoc device. In: International Conference on Brain Informatics. December 4-7, Macao, China, 2012; p. 175-184.

15. Pham TD, Tran D. Emotion recognition using the emotiv epoc device. In: International Conference on Neural Information Processing. 12-15 November, Doha, Qatar, 2012; p. 394-399.

16. Lu Z, Chen X, Li Q, Zhang X, Zhou P. A hand gesture recognition framework and wearable gesture-based interaction prototype for mobile devices. IEEE transactions on human-machine systems. 2014; 44(2): 293-299.

17. Milica Djurić-Jovičić. Inertial Sensors Signal Processing Methods For Gait Analysis Of Patients With Impaired Gait Patterns, PhD thesis, University of Belgrade - School of Electrical Engineering, Belgrade, 2012.

18. Torniainen J, Cowley B, Henelius A, Lukander K, Pakarinen S. Feasibility of an electrodermal activity ring prototype as a research tool. In: 37 th Annual International Conference of the Engineering in Medicine and Biology Society (EMBC), 25-29 August, Milano, Italy, 2015; p. 6433-6436.

19. Suoja K, Liukkonen J, Jussila, J, Salonius H, Venho N, Sillanpää V et al. Application for pre-processing and visualization of electrodermal activity wearable data. In: EMBEC \& NBC. 11-15 June, Tampere, Finland, 2017; p. 93-96.

20. Bulling A, Gellersen H. Toward mobile eye-based human-computer interaction. IEEE Pervasive Computing, 2010; 9(4): 8-12.

21. Cappon G, Acciaroli G, Vettoretti M, Facchinetti A. Sparacino G. Wearable Continuous Glucose
Monitoring Sensors: A Revolution in Diabetes Treatment. Electronics. 2017; 6(3): 65.

22. Zheng YL, Yan BP, Zhang YT, Poon CC. An armband wearable device for overnight and cuff-less blood pressure measurement. IEEE transactions on biomedical engineering. 2014; 61(7): 2179-2186.

23. Hayashi C. What is Data Science? Fundamental Concepts and a Heuristic Example. In: Data Science, Classification, and Related Methods. Studies in Classification, Data Analysis, and Knowledge Organization. Toky: Springer; 1998.

24. Bishop C. Pattern recognition and machine learning. Berlin: Springer; 2006.

25. Goodfellow I, Bengio Y, Courville A. Deep Learning. Cambridge:The MIT Press; 2016.

26. Chin-Teng L, George Lee CS. Feedforward neural networks - Neural Fuzzy Systems: A Neuro-Fuzzy Synergism to Intelligent Systems. New York: Prentice Hall; 1996.

27. Krizhevsky A, Sutskever I, Hinton GE. Imagenet classification with deep convolutional neural networks. In: Advances in neural information processing systems. 3-8 December, Lake Tahoe, Harrahs and Harveys, 2012; p. 1097-1105.

28. Lipton ZC, Berkowitz J, Elkan C. A critical review of recurrent neural networks for sequence learning. arXiv preprint arXiv:1506.00019. 2015.

29. Redmond SJ, Lovell NH, Yang GZ, Horsch A, Lukowicz P, Murrugarra L, Marschollek M. What does big data mean for wearable sensor systems?: Contribution of the IMIA wearable sensors in healthcare WG. Yearbook of medical informatics, 2014; 9(1): 135-142.

30. Hansen MM, Miron-Shatz T, Lau AYS, Paton C. Big data in science and healthcare: a review of recent literature and perspectives: contribution of the IMIA social media working group. Yearbook of medical informatics, 2014; 9(1): 21-26.

31. Andreu-Perez J, Leff DR, Ip HM, Yang GZ. From wearable sensors to smart implants--toward pervasive and personalized healthcare. IEEE Transactions on Biomedical Engineering. 2015; 62(12): 2750-2762.

32. Simpao AF, Ahumada LM, Gálvez JA, Rehman MA. A review of analytics and clinical informatics in health care. Journal of medical systems. 2014; 38(4): 1-7.

33. Gravina R, Alinia P, Ghasemzadeh H, Fortino G. Multi-sensor fusion in body sensor networks: Stateof-the-art and research challenges. Information Fusion. 2017; 35: 68-80.

34. Banaee H, Ahmed MU, Loutfi A. Data mining for wearable sensors in health monitoring systems: a review of recent trends and challenges. Sensors. 2013; 13(12): 17472-17500.

35. Sarkar S, Reddy K, Dorgan A, Fidopiastis C, Giering $M$. Wearable EEG-based activity recognition in PHM-related service environment via deep learning. Int. J. Progn. Health Manag, 2016; 7: 1-10.

36. Längkvist $M$, Karlsson L, Loutfi A. Sleep stage 
classification using unsupervised feature learning. Advances in Artificial Neural Systems. 2012;2012:1-9.

37. Zhang J, Wu Y, Bai J, Chen F. Automatic sleep stage classification based on sparse deep belief net and combination of multiple classifiers. Transactions of the Institute of Measurement and Control. 2016; 38: 435-451.

38. Dong H, Supratak A, Pan W, Wu C, Matthews PM, Guo Y. Mixed neural network approach for temporal sleep stage classification. IEEE Transactions on Neural Systems and Rehabilitation Engineering. 2018; 26: 324-333.

39. Wulsin D, Gupta J, Mani R, Blanco J, Litt B, Modeling electroencephalography waveforms with semi-supervised deep belief nets: fast classification and anomaly measurement. Journal of neural engineering. 2011; 8(3): 1-28.

40. Tabar YR, Halici U. A novel deep learning approach for classification of EEG motor imagery signals. Journal of neural engineering. 2016; 14:016003.

41. Lu N, Li T, Ren X, Miao H. A deep learning scheme for motor imagery classification based on restricted boltzmann machines. IEEE transactions on neural systems and rehabilitation engineering. 2017; 25: 566-576.

42. Ma T, Li H, Yang H, Lv X, Li P, Liu T, et al. The extraction of motion-onset VEP BCI features based on deep learning and compressed sensing. Journal of neuroscience methods. 2017; 275: 80-92.

43. Atzori M, Cognolato M, Müller H. Deep learning with convolutional neural networks applied to electromyography data: a resource for the classification of movements for prosthetic hands. Frontiers in neurorobotics. 2016; 10(9): 1:8.

44. Atzori M, Gijsberts A, Heynen S, Hager AGM, Deriaz O, Van Der Smagt et al. Building the Ninapro database: A resource for the biorobotics community. In: 4th IEEE RAS \& EMBS International Conference on Biomedical Robotics and Biomechatronics (BioRob). 24-27 June, Rome, Italy, 2012; p. 1258-1265.

45. Yan Y, Qin X, Wu Y, Zhang N, Fan J, Wang L. A restricted Boltzmann machine based two-lead electrocardiography classification. In: 12th International Conference on Wearable and Implantable Body Sensor Networks (BSN). 9-12 June, Cambridge, Massachusetts, 2015; p. 1-9.

46. Goldberger AL, Amaral LA, GlassL, Hausdorff JM, Ivanov PC, Mark RG et al. Physiobank, physiotoolkit, and physionet: components of a new research resource for complex physiologic signals. Circulation. 2000; 101(23): e215-e220.

47. Ripoll VJR, Wojdel A, Romero E, Ramos P, Brugada J. ECG assessment based on neural networks with pretraining. Applied Soft Computing, 2016; 49: 399-406.

48. Page A, Kulkarni, A, Mohsenin T, Utilizing deep neural nets for an embedded ECG-based biometric authentication system. In: Biomedical Circuits and Systems Conference (BioCAS), 22-24 October,
Atlanta, GA, USA, 2015. p. 1-4.

49. Ashiquzzaman A, Tushar AK, Islam MR, Shon D, Im K, Park JH et al. Reduction of Overfitting in Diabetes Prediction Using Deep Learning Neural Network. In: IT Convergence and Security 2017. Singapore: Sringer; 2018; p. 35-43.

50. Shashikumar SP, Shah AJ, Li Q, Clifford GD, Nemati $S$, A deep learning approach to monitoring and detecting atrial fibrillation using wearable technology. In: IEEE EMBS International Conference of Biomedical \& Health Informatics (BHI), 4-7 March, Las Vegas, Nevada, USA, p. 141-144.

51. Jindal V, Birjandtalab J, Pouyan MB, Nourani M, An adaptive deep learning approach for PPG-based identification. In: 38th Annual International Conference of the Engineering in Medicine and Biology Society (EMBC), 17-20 August, Lake Buena Vista, Orlando, USA, 2016. p. 6401-6404.

52. Ruiz-Rodríguez JC, Ruiz-Sanmartín A, Ribas V, Caballero J, García-Roche A, Riera J et al. Innovative continuous non-invasive cuffless blood pressure monitoring based on photoplethysmography technology. Intensive care medicine. 2013; 39(9): 1618-1625.

53. Ordóñez FJ, Roggen D. Deep convolutional and lstm recurrent neural networks for multimodal wearable activity recognition. Sensors. 2016; 16(1): 1-25.

54. Saeedi R, Norgaard S, Gebremedhin AH. A closed-loop deep learning architecture for robust activity recognition using wearable sensors. In: IEEE International Conference on Big Data. 11-14 December, Boston, MA, USA, 2017; p. 473-479.

55. Bhattacharya S, Lane ND. From smart to deep: Robust activity recognition on smartwatches using deep learning. In: IEEE International Conference on Pervasive Computing and Communication Workshops (PerCom Workshops). 14-18 March, Sydney, Australia, 2016; pp. 1-6.

56. Ronao CA, Cho SB. Human activity recognition with smartphone sensors using deep learning neural networks. Expert Systems with Applications. 2016; 59: 235-244.

57. Ravi D, Wong C, Lo B, Yang GZ. cs. In: 13th International Conference on Wearable and Implantable Body Sensor Networks (BSN). 14-17 June, San Francisco, CA, USA, 2016; p. 71-76.

58. Eskofier BM, Lee SI, Daneault JF, Golabchi FN, Ferreira-Carvalho G, Vergara-Diaz G. et al. Recent machine learning advancements in sensor-based mobility analysis: deep learning for Parkinson's disease assessment. In: IEEE 38th Annual International Conference of the Engineering in Medicine and Biology Society (EMBC). 17-20 August, Lake Buena Vista, Orlando, USA, 2016; p. 655-658.

59. Ravì D, Wong C, Deligianni F, Berthelot M, AndreuPerez J, Lo B, Yang GZ. Deep learning for health informatics. IEEE journal of biomedical and health informatics, 2017; 21(1): 4-21.

60. Miotto R, Wang F, Wang S, Jiang X, Dudley JT. Deep 
Janković M. et al. Deep learning approaches for human activity recognition using wearable technology. MedPodml 2018, 69(3):14-24

learning for healthcare: review, opportunities and challenges. Briefings in bioinformatics. 2017; 1-11.

61. Faust O, Hagiwara Y, Hong TJ, Lih OS, Acharya UR. Deep learning for healthcare applications based on physiological signals: a review. Computer methods and programs in biomedicine. 2018; 1-31.

62. Lane ND, Georgiev P. Can deep learning revolutionize mobile sensing? In: Proceedings of the 16th International Workshop on Mobile Computing Systems and Applications. 12-13 February, Santa Fe, NM, USA, 2015; p. 117-122.

63. Yao S, Hu S, Zhao Y, Zhang A, Abdelzaher T.
Deepsense: A unified deep learning framework for time-series mobile sensing data processing. In: Proceedings of the 26th International Conference on World Wide Web. 3-7 April, Perth, Australia, 2017; p. 351-360.

64. Lane ND, Bhattacharya S, Georgiev P, Forlivesi C, Jiao L, Qendro L, Kawsar F. Deepx: A software accelerator for low-power deep learning inference on mobile devices. In: 15th ACM/IEEE International Conference on Information Processing in Sensor Networks (IPSN). 11-14 April, Vienna, Austria, 2016; p. 1-12. 